\title{
A RANDOMIZED CONTROLLED COMPARISON OF ZOLIDRONATE TO PAMIDRONATE EFFECTS UPON BONE MINERALIZATION AND FRACTURE INCIDENCE IN CHILDREN WITH SEVERE OSTEOGENESIS IMPERFECT
}

\author{
S. Salehpour ${ }^{1}$, S. Tavakoli ${ }^{2}$ \\ ${ }^{1}$ Pediatric Endocrinology, Shahid Beheshti University of Medical Sciences(SBMU), ${ }^{2}$ Pediatric Orthopedics, \\ Tehran University of Medical Sciences, Tehran, Iran
}

Background: Severe osteogenesis imperfect (OI) is a disorder characterized by osteopenia, frequent fractures, progressive deformities, loss of mobility, and chronic bone pain.

Objective: The main objective of this study was to compare the efficacy and safety of zolidronate with those of pamidronate on bone mineralization and fracture incidence in OI.

Methods: The study was performed at the outpatient pediatric endocrine clinic of a children hospital. 27 prepubertal children (2-11 years old) with severe OI divide randomly in two groups, treated with either zolidronate (13 patients) or pamidronate (14 patients) for 2 years. The main outcome measures were bone mineral density and fracture incidence. Secondarily, their effects on mobility and bone turnover markers (alkaline phosphatase and C- telopeptide) were compared.

Result: Zolidronate and pamidronate had the same effects on improving the bone mineral density $(\mathrm{p}<0.001)$ and reducing the fracture incidence $(\mathrm{p}<0.001)$. Their effects on mobility and bone turnover markers were also similar.

Conclusion: The efficacy of zolidronate and pamidronate on improving bone mineralization and reducing fracture incidence and their safety in children with severe OI are the same. 